\title{
Effect of bilayer number on the photoluminescent property of TPE-based self-assembled film
}

\author{
CHEN DiDi ${ }^{1}$, FENG Xiao ${ }^{1}$, GU ShangZhi ${ }^{1}$, TONG Bin ${ }^{1}$, SHI JianBing ${ }^{1}$, ZHI JunGe $^{2}$ \& \\ DONG YuPing ${ }^{1 *}$
}

${ }^{1}$ College of Materials Science and Engineering, Beijing Institute of Technology, Beijing 100081, China;

${ }^{2}$ College of Chemistry, Beijing Institute of Technology, Beijing 100081, China

Received April 3, 2013; accepted May 6, 2013; published online June 27, 2013

\begin{abstract}
Tetraphenylethylene (TPE) derivatives have been proved to be typical aggregation-induced emission (AIE) luminogens when they were aggregated in the free three-dimensional space. In order to reveal the effect of the dimensional degree on AIE property of TPEs, we utilized tetra(4-hydroxyphenyl)ethylene (TPE-4OH) and 1,4-benzenediamine diazonium salt (BD) to fabricate the ultrathin films (TPE-4OH/BD LBL SA film) through layer-by-layer self-assembled technique. The interaction between TPE-4OH and $\mathrm{BD}$ in the films was converted from electrostatic force and hydrogen-bond to covalent bonds through photodecomposition of diazonium groups under UV irradiation. Fluorescence emission spectroscopy, UV-Vis absorption spectroscopy and atomic force microscope were carried out to evaluate the relationship between bilayer number and photoluminescence of the TPE-based self-assembled films. The experimental results showed that the TPE-based film with three bilayers only displayed AIE character, whereas the fluorescence of the film became randomly changed if the bilayer number was above three. It is supposed that the fluorescence property of the TPE-4OH/BD LBL SA film with limited molecular length in $z$-axis and infinite aggregation space in $x$ - and $y$-axis is dominated by two competitive effects, one is the partial restriction of intramolecular rotation through short intermolecular interactions in cross-linked structure of TPE-4OH/BD, and the other is deactivation of its excited state through unrestricted intramolecular rotations or $\pi-\pi$ interactions.
\end{abstract}

tetraphenylethylene, aggregation-induced emission, layer-by-layer self-assembled film, 1,4-benzenediamine diazonium salt

Citation: $\quad$ Chen D D, Feng X, Gu S Z, et al. Effect of bilayer number on the photoluminescent property of TPE-based self-assembled film. Chin Sci Bull, 2013, 58: 2728-2732, doi: 10.1007/s11434-013-5943-7

Recently, there has been a surge in the efforts directed to the development of luminogens that can emit more efficiently in solid state than in solutions [1-7]. This unique photophysical phenomenon was first reported by Tang's group [8] in 2001. "Aggregation-induced emission (AIE)" [8-10], as he called it, is exactly opposite to the aggregation-caused quenching (ACQ) effect. AIE-active molecules are nonemissive luminogens when molecularly dissolved in their good solvents but induced to emit efficiently by aggregate formation, mainly owing to the restricted intramolecular rotations (RIR) [11,12].

Tetraphenylethylene (TPE) as an iconic AIE luminogens

\footnotetext{
*Corresponding author (email: chdongyp@ bit.edu.cn)
}

has a potential utility in sensors and organic lightingemitting materials [13-18]. In TPE dilute solutions, dynamic and nonradiative rotation of the phenyl rings deactivates its excited states. According to literatures, the fluorescence is permitted to deactivation by obstructing the rotation of the phenyl groups through short intermolecular interactions in TPE aggregates growing in all three dimensions [19-21]. However, the photoluminescent property is not well explored if the growth of TPE aggregates is limited in certain dimensions. To fill this blank, we proposed two questions from the microscopic view: what is the relationship between the limited aggregate manner and photoluminescent property of TPE, and what is the key factor in dominating this relationship? 
The self-assembly technique that can prepare homogeneous and stable film with limited growing directions is utilized to study above questions. It is based on the electrostatic attraction of oppositely charged polyelectrolytes and was developed by Decher and coworkers [22-24], who extended the pioneering work of Iler [25]. Because of simple in procedure, easy control of the structures, thickness, and composition at the molecular level, and friendly to the environment [22,26], it has become more and more attractive and has been successfully applied to the preparation of advanced membranes with desired components and structure.

Herein, we reported a vertical growth of layer-by-layer self-assembled (TPE-4OH/BD LBL SA) film based on tetra(4-hydroxyphenyl)ethylene and 1,4-benzenediamine diazonium salt. The growth of TPE moieties in the film was restricted in one dimension ( $z$-axis) and its aggregating was not limited in the rest of two dimensions ( $x$ - and $y$-axis). The effect of restricted aggregation on the photoluminescence of TPE-based compound in the solid state was studied. Fluorescence emission spectroscopy, UV-Vis absorption spectroscopy, and atomic force microscope (AFM) were used to observe the relationship between the bilayer number and photoluminescent property of films on the substrates.

TPE-4OH was prepared via McMurry Olefination reaction from 4,4-dihydroxybenzophenone (Scheme 1) [27]. BD was synthesized from 1,4-diaminobenzene according to ref. [3]. 4,4-Dihydroxybenzophenone and 1,4-diaminobenzene were purchased from Aladdin and Sinopharm, respectively. Tetrahydrofuran (THF) was dried over and distilled from $\mathrm{Na}$ and benzophenone under an atmosphere of dry nitrogen. Other reagents were purchased from Beijing Chemical Reagent Co. and used as received without further purification.

UV-Vis absorption spectra were recorded on a TU-1901 UV-Vis Spectrophotometer (Beijing Purkinje General Instrument Co., Ltd.). Fluorescence measurements were carried out with a Hitachi F-7000 Fluorescence Spectrophotometer. The surface structure of TPE-4OH/BD LBL SA film was observed by a Bruker Dimension Icon AFM.

The procedures for the pretreatment of quartz slide were described in ref. [28]. In a dark environment, a pretreated quartz slide was firstly immersed into a solution of BD $(0.5$ $\mathrm{mg} / \mathrm{mL}$ ) for $10 \mathrm{~min}$, then rinsed with deionized water thoroughly, and dried by flowing air in room temperature. Subsequently, it was immersed in a suspension of TPE-4OH $\left(0.5 \mathrm{mg} / \mathrm{mL}\right.$, in $1.0 \mathrm{wt} \% \mathrm{NaHCO}_{3}$ solution) for $10 \mathrm{~min}$, then

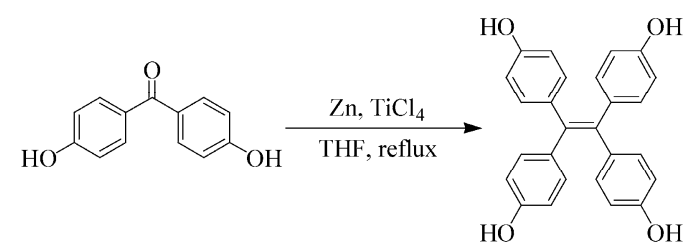

Scheme 1 Synthesis route of TPE-4OH via McMurry Olefination reaction. rinsed and dried in room temperature again, at last UV irradiation $\left(230 \mu \mathrm{W} / \mathrm{cm}^{2}, 360 \mathrm{~nm}\right)$ for $7 \mathrm{~min}$. Finally, it was immersed in deionized water and dispersed for 1 min using ultrasound. Thus, a one-bilayer TPE-4OH/BD LBL SA film was formed.

UV-Vis spectroscopy was used to monitor the formation of multilayer prepared by LBL SA process (Figure 1). The maximum absorption peak at about $280 \mathrm{~nm}$ was attributed to the characteristic absorption of diazonium group. The UV absorbance increased with increasing of bilayer number, indicating $\mathrm{BD}$ is employed as the stable anchoring layer between the quartz slide and TPE-4OH through electrostatic interaction and hydrogen-bonding interaction by the LBL SA technique, respectively. The quantity of TPE-4OH and BD increased with the bilayer number increasing. After irradiation of UV light, the film was further stabilized by means of converting the electrostatic interaction to covalent bond (Scheme 2). As shown in Figure 1, the relationship between the absorbance and the number of bilayers revealed that the amounts of deposition of each bilayer were equal. The results demonstrated the successful fabrication of TPE4OH/BD multilayer self-assembled films on substrate.

TPE-4OH hardly shows any fluorescence in its good solvents. Thus, the increment of fluorescence intensity in the first three bilayers (Figure 2) was attributed to the AIE effect. However, the fluorescence intensity randomly changed from the fourth bilayer, although the rule of variations remained same in another five repeated experiments. We assumed that in the first three bilayers, TPE-4OH/BD self-assembled film was regularly aligned to the substrate with fewer configurations. When the bilayer number was over four, the molecular configuration number was sharply increased with the growth of molecular chains, and TPE-4OH could no longer retain tight arrangement. That is, the intramolecular rotations cannot be completely restricted by the only limitations in $x$ - and $y$-axis dimensions if the molecular chains exceed a particular length and the limitations in $z$-axis dimension does not exist. It is supposed that the random changes of fluorescence intensities were due to the competition of AIE and ACQ effect.

In order to further validate the effect of the limitation of dimensional degree on the AIE property of TPE-based compounds, the fluorescence and UV-Vis spectra of the mixture of TPE-4OH suspension and BD solution were measured before and after irradiation of UV light. The fluorescence intensity sharply increased (Figure 3 (a)) while intensity of UV absorption peak decreased after irradiation of UV light (Figure 3(b)). The enhancement of the fluorescence intensity was resulted from the further restriction of intramolecular rotations of TPE moieties in the photosynthetic product of TPE-4OH/BD in an unfettered threedimensional space. In contrast, the fluorescence property of TPE-4OH/BD LBL SA films was simultaneously dominated by RIR and ACQ process when the growth of molecular chains was limited in one dimension (z-axis) and the assem- 

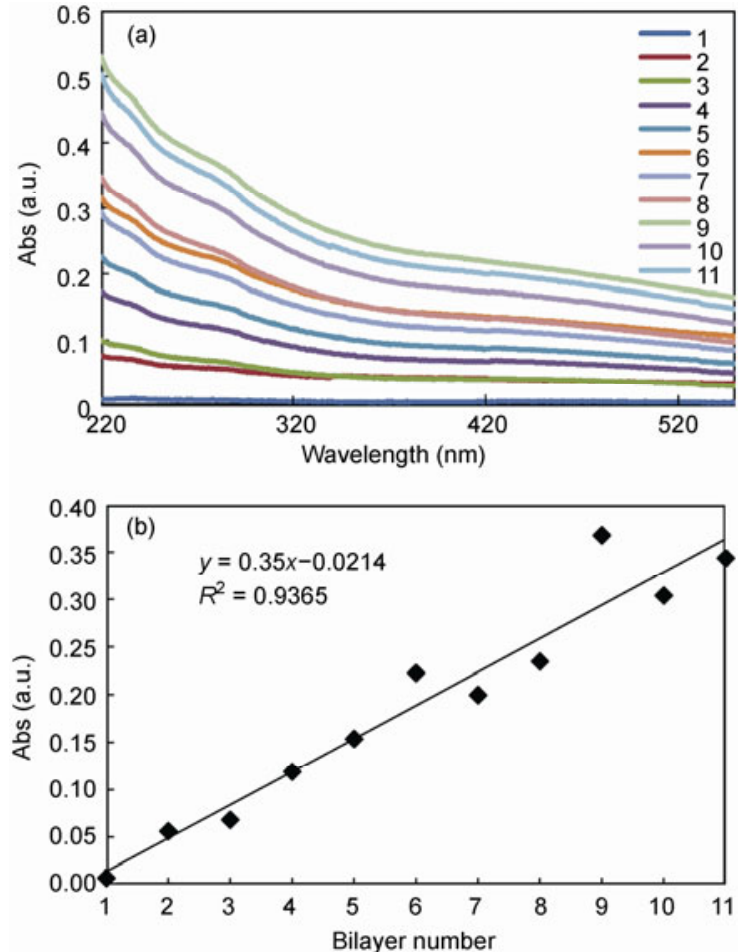

Figure 1 UV-Vis spectra of TPE-4OH/BD LBL SA film. (a) UV-Vis spectra with different bilayer numbers. (b) The UV absorbance intensity changes with different bilayer numbers at $280 \mathrm{~nm}$.
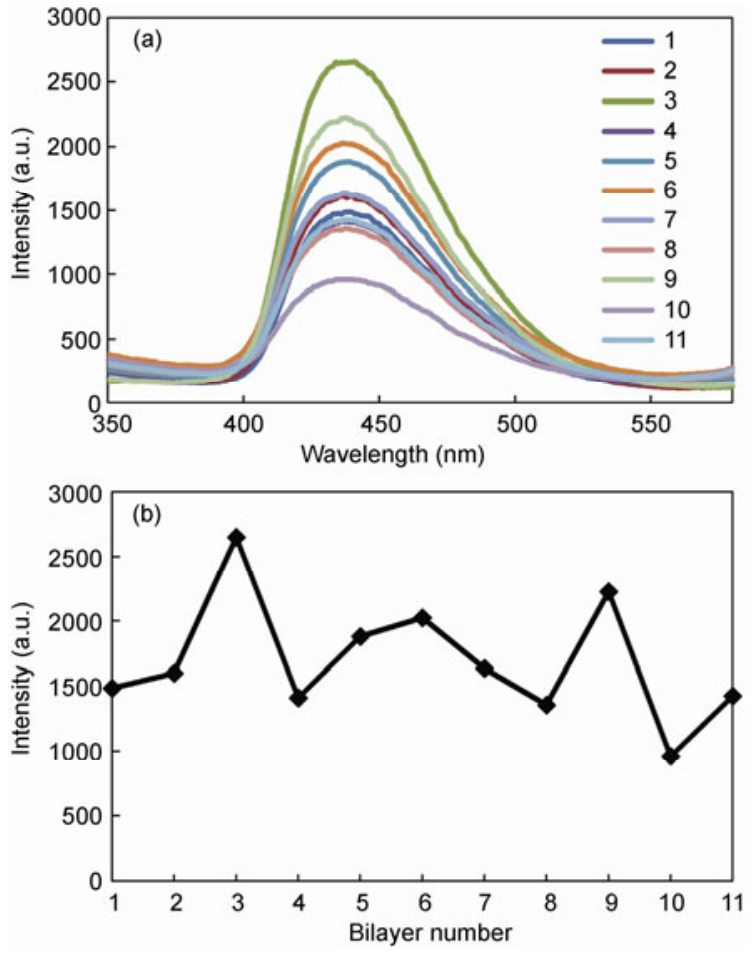

Figure 2 Fluorescence spectra of TPE-4OH/BD LBL SA film. (a) The fluorescence spectra with different bilayer numbers. (b) Fluorescence intensity changes with different bilayer numbers at $438 \mathrm{~nm}$.
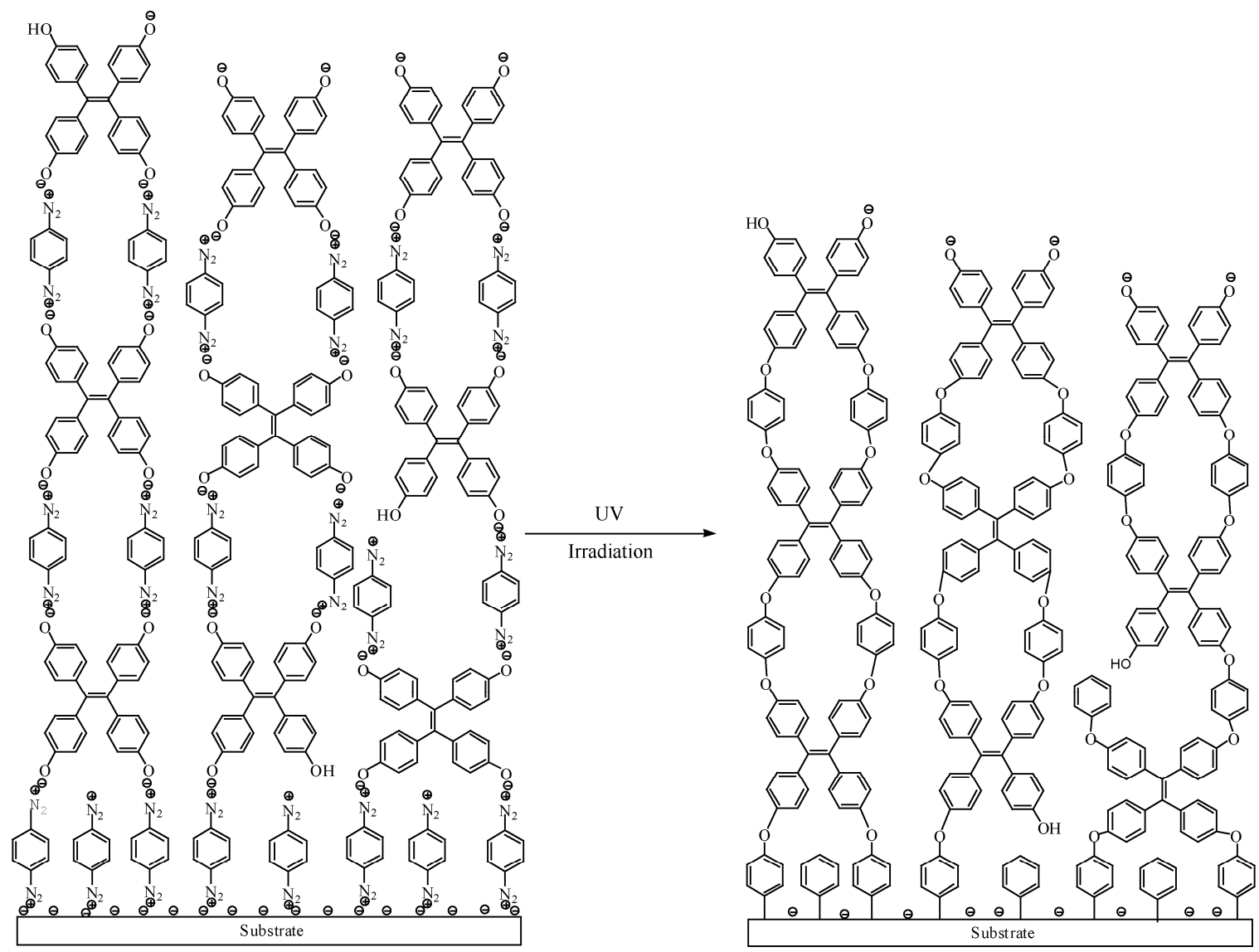

Scheme 2 Diagram of TPE-4OH/BD LBL SA film. 
bling was not limited in another two dimensions ( $x$ and $y$ axes) in the case of LBL films.

In order to further investigate the reasons behind the fluorescence changings along with varying the bilayer numbers, AFM was used to observe the surface morphologies of TPE-4OH/BD LBL SA films. Surface topography (left) and phase images (right) of the films with three- and four- bilayer were shown in Figure 4. Average surface roughness values ( $\mathrm{Ra}$ ) measured from the topography images in $2 \mu \mathrm{m}$ $\times 2 \mu \mathrm{m}$ regions were as follows: 1.15 and $2.16 \mathrm{~nm}$ for three-bilayer and four-bilayer film, respectively. It revealed that three-bilayer film has more regular aggregating structure. At the same time, the surface of three-bilayer selfassembled film was obvious flatter than that of four-bilayer
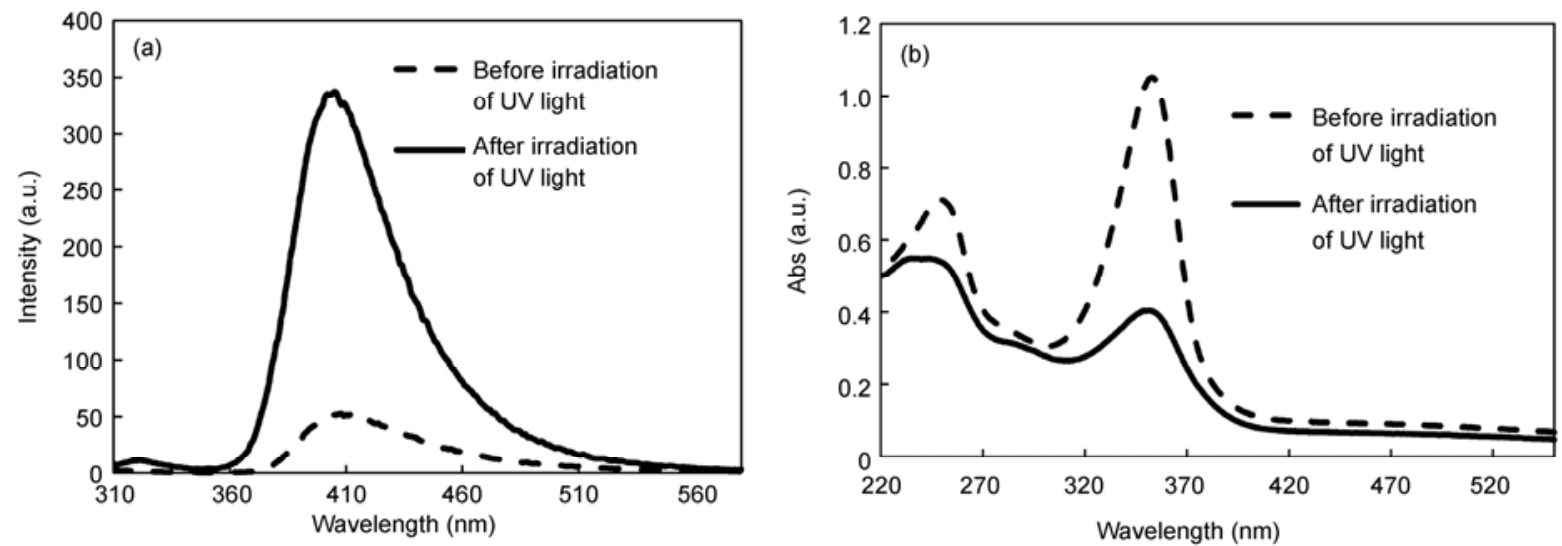

Figure 3 (a) Fluorescence spectra and (b) UV-Vis spectra of the $1: 1$ (v/v) mixture of TPE- $4 \mathrm{OH}\left(0.5 \mathrm{mg} / \mathrm{mL}\right.$, in $1.0 \mathrm{wt} \% \mathrm{NaHCO}_{3}$ solution) suspension and $\mathrm{BD}(0.5 \mathrm{mg} / \mathrm{mL})$ solution.
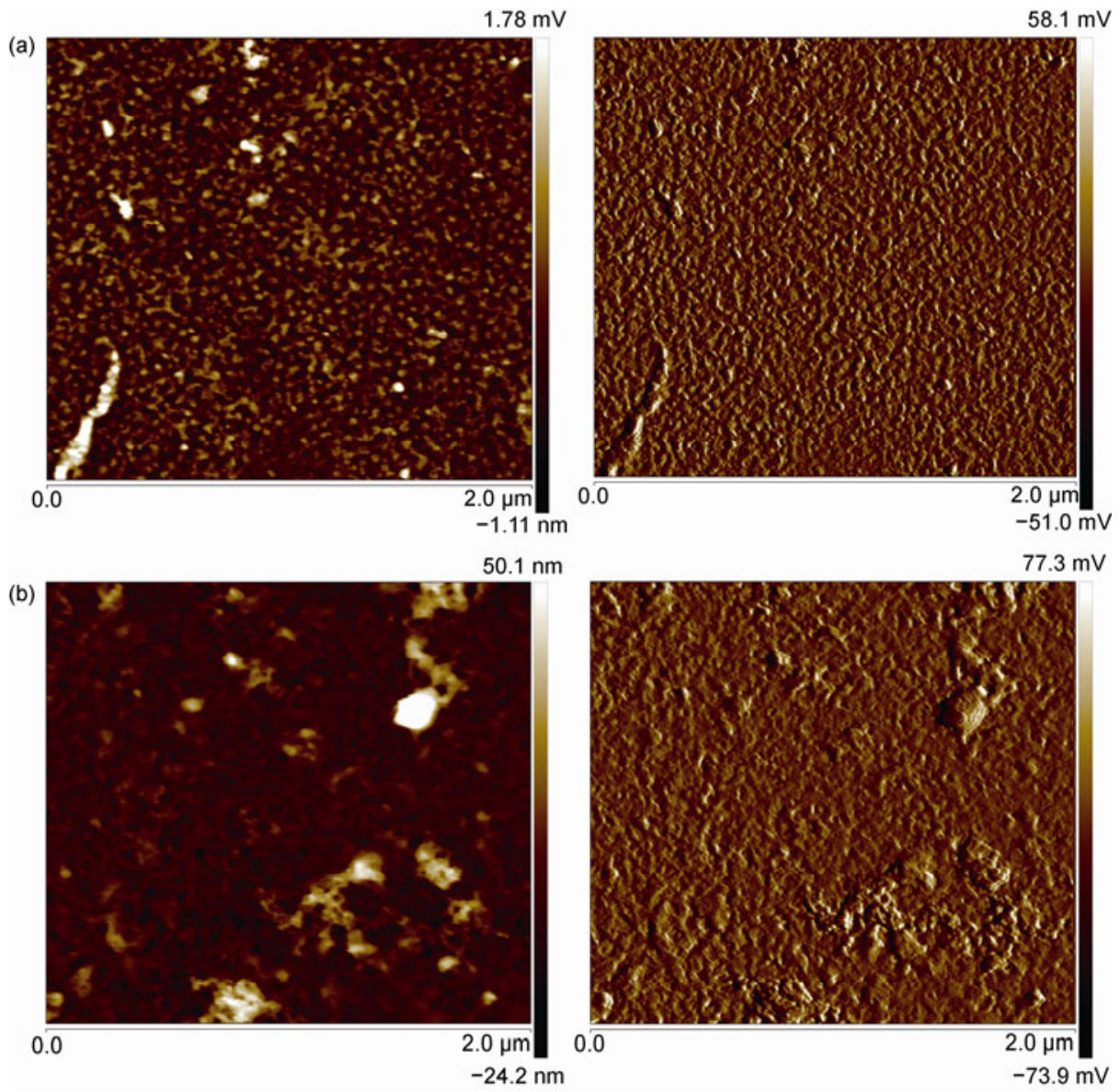

Figure 4 AFM images of a $2 \mu \mathrm{m} \times 2 \mu \mathrm{m}$ region. (a) three-bilayer; (b) four-bilayer. Topography of each film is shown in the left panels and the corresponding phase images in the right panels. 
ones (Figure 4, right panels). Compared with Figure 4 (b), a rather homogeneous structure was showed in Figure 4 (a). These observations were consistent with the fluorescence spectra shown in Figure 2 and supported the above explanations. That is, if the molecular chains exceeded a certain length, the configuration of the aggregate structure of TPE-based compounds only formed in a two-dimensional space would become more random and thus can not completely restrict intramolecular rotations of TPE and restrain the nonradiative energy conversion. So that, AIE property was not visualized when the bilayer number was above three.

The TPE-4OH/BD LBL SA films were successfully fabricated through electrostatic interaction, which was further converted to covalent bonds via photodecomposition of diazonium groups under UV irradiation. UV analytical results showed the amount of each layer of TPE-4OH/BD LBL SA film was almost equal. Fluorescence spectra and AFM, however, indicated that the TPE-based films with three bilayers or less displayed AIE character due to the regular arrangement of the aggregate structures. When bilayer number is over three, the photoluminescence of films could not sustainedly increase. It is attributed to the noneffective restriction of intramolecular rotations in the irregular aggregate structure of TPE-4OH/BD, which could only assemble in two dimensions ( $x$ and $y$ axes). That is, the TPEbased compounds need aggregating in all three dimensions to exhibit AIE characters.

This work was supported by the National Natural Science Foundation of China (51073026, 51061160500, 20944004, 21074011, 21004004), the National Basic Research Program of China (2013CB834704), and the Special- ized Research Fund for the Doctoral Program of Higher Education of China (20091101110031).

1 Shi J, Wu Y, Sun S, et al. J Polym Sci Part A: Polym Chem, 2013, 51: 229-240

2 Wu Y, Shi J, Tong B, et al. Acta Polym Sin, 2012, 12: 1482-1490

3 Zhang Y, Xia J, Feng X, et al. Sen Actuators B: Chem, 2012, 161: 587-593

4 Li C, Wu T, Hong C, et al. Angew Chem Int Ed, 2012, 51: 455-459

Aldred M, Li C, Zhu M. Chem Eur J, 2012, 18: 16037-16045

Wang Y, Liu T, Bu L, et al. J Phys Chem C, 2012, 116: 15576-15583

Wu W, Ye S, Tang R, et al. Polymer, 2012, 53: 3163-3171

8 Luo J, Xie Z, Lam J W Y, et al. Chem Commun, 2001, 18: $1740-1741$

9 Qin A, Zhang Y, Han N, et al. Sci China Chem, 2012, 55: 772-778

10 Li H, Mei J, Wang J, et al. Sci China Chem, 2011, 54: 611-616

11 Huang J, Yang X, Wang J, et al. J Mater Chem, 2012, 22: 2478-2484

12 Luo X, Zhao W, Shi J, et al. J Phys Chem C, 2012, 116: 2196721972

13 Sun F, Zhang G, Zhang D. Chin Sci Bull, 2012, 57: 4284-4288

14 Wang J, Mei J, Zhao E, et al. Macromolecules, 2012, 45: 7692-7703

15 Wang J, Mei J, Hu R, et al. J Am Chem Soc, 2012, 134: 9956-9966

16 Liu Y, Yu Y, Lam J, et al. Chem Eur J, 2010, 16: 8433-8438

17 Jin J, Chen X, Liu Y, et al. Acta Polym Sin, 2011, 9: 1079-1085

18 Zhao Z, Liu D, Lam J, et al. Sci China Chem, 2010, 53: 2311-2317

19 Feng X, Tong B, Shen J, et al. J Phys Chem B, 2010, 114: 1673116736

20 Qian L, Tong B, Shen J, et al. J Phys Chem B, 2009, 113: 9098-9103

21 Yuan W Z, Mahtab F, Gong Y, et al. J Mater Chem, 2012, 22: 10472-10479

22 Decher G. Science, 1997, 277: 1232-1237

23 Decher G, Hong J D, Bunsen-Ges B. Phys Chem, 1991, 95: 14301434

24 Decher G, Hong J D, Schmitt J. Thin Solid Films, 1992, 210: 831835

25 Iler R K. J Colloid Interface Sci, 1966, 21: 564-569

26 Mao G, Tsao Y H, Tirrell M, et al. J Surface Colloid, 1995, 11: 942952

27 Wu Y, Shi J, Tong B, et al. Acta Polym Sin, 2012, 4: 453-461

28 Sun J, Wu T, Sun Y, et al. Chem Commun, 1998, 70: 1853-1854

Open Access This article is distributed under the terms of the Creative Commons Attribution License which permits any use, distribution, and reproduction in any medium, provided the original author(s) and source are credited. 